\title{
Article \\ Association between Perceived Stress and Rhinitis-Related Quality of Life: A Multicenter, Cross-Sectional Study
}

\author{
Il Gyu Kong ${ }^{1}\left(\mathbb{D}\right.$, Chae-Seo Rhee ${ }^{2,3,4,5}$, Jung Woo Lee ${ }^{2}$, Hyojin Yim ${ }^{2}$, Min Jung Kim ${ }^{6}$, Yunhee Choi ${ }^{6}$, \\ Doo Hee Han $2, * \mathbb{B}$ and on behalf of the Allergic Rhinitis Cohort Study Group ${ }^{+}$ \\ 1 Department of Otorhinolaryngology, Hospital Medicine Center, Seoul National University Hospital, \\ Seoul 03080, Korea; yeah19@snuh.org \\ 2 Department of Otorhinolaryngology, Seoul National University College of Medicine, \\ Seoul National University Hospital, Seoul 03080, Korea; csrhee@snu.ac.kr (C.-S.R.); \\ jwooya83@naver.com (J.W.L.); byeolalae@naver.com (H.Y.) \\ 3 Graduate School of Immunology, Seoul National University College of Medicine, Seoul 03080, Korea \\ 4 Institute of Allergy and Clinical Immunology, Seoul National University Biomedical Research Center, \\ Seoul 03080, Korea \\ 5 Sensory Organ Research Institute, Seoul National University Biomedical Research Center, Seoul 03080, Korea \\ 6 Medical Research Collaborating Center, Seoul National University Hospital, Seoul 03080, Korea; \\ kmj1094@naver.com (M.J.K.); yhchoi@snuh.org (Y.C.) \\ * Correspondence: handh@snu.ac.kr; Tel.: +82-2-2072-4038 \\ + Membership of the Allergic Rhinitis Cohort Study Group is provided in Acknowledgements.
}

check for updates

Citation: Kong, I.G.; Rhee, C.-S.; Lee, J.W.; Yim, H.; Kim, M.J.; Choi, Y.; Han, D.H.; on behalf of the Allergic

Rhinitis Cohort Study Group.

Association between Perceived Stress and Rhinitis-Related Quality of Life: A Multicenter, Cross-Sectional Study. J. Clin. Med. 2021, 10, 3680. https:// doi.org/10.3390/jcm10163680

Academic Editor: Alfonso Del Cuvillo

Received: 24 May 2021

Accepted: 13 August 2021

Published: 19 August 2021

Publisher's Note: MDPI stays neutral with regard to jurisdictional claims in published maps and institutional affiliations.

Copyright: (C) 2021 by the authors Licensee MDPI, Basel, Switzerland. This article is an open access article distributed under the terms and conditions of the Creative Commons Attribution (CC BY) license (https:// creativecommons.org/licenses/by/ $4.0 /)$.
Abstract: Allergic rhinitis (AR), a common chronic disease, impairs patients' quality of life (QoL). This study aimed to determine the effect of perceived stress on disease-related QoL in AR patients. There were 741 patients from eight medical centers of the Allergic Rhinitis Cohort (ARCO) study. Data on sociodemographics, chronic conditions, AR severity, perceived stress level and Rhinoconjunctivitis QoL Questionnaire (RQLQ) results, and laboratory test findings were collected. The relationship between perceived stress and total RQLQ was analyzed using multiple linear regression. Potential confounding variables were adjusted. A high perceived stress level was associated with a high total RQLQ, which reflected worsening disease-related QoL. The high stress level was associated with an increased total RQLQ of 1.210 (95\% confidence interval, $0.831-1.589 ; p<0.0001$ ) compared with the very low level. In the final model, the multiple regression-adjusted $\mathrm{R}^{2}$ for RQLQ in AR participants was 0.5279 , and perceived stress levels contributed $4.08 \%$ in additional explanatory power to RQLQ in AR patients. In conclusion, perceived stress is a potentially modifiable risk factor for decreased disease-related QoL in patients with AR, which may be improved with stress management.

Keywords: allergic rhinitis; stress; disease-related quality of life; cross-sectional study; rhinoconjunctivitis quality of life questionnaire; stress management; Allergic Rhinitis Cohort

\section{Introduction}

Allergic rhinitis (AR) is one of the most common chronic diseases with a high global burden. It is characterized by an IgE-mediated immune reaction to allergens. In a European multicenter study [1], the age- and sex-standardized prevalence of AR in people aged 20 to 44 years ranged from $11.8 \%$ to $46.0 \%$. In the International Study of Asthma and Allergy in Childhood (ISAAC) [2], AR prevalence increased from $8.5 \%$ in individuals aged 6 to 7 years to $14.6 \%$ in those aged 13 to 14 years. The 2010 Korea National Health and Nutrition Examination Survey [3] reported that the overall prevalence of AR based on laboratory tests was $16.2 \%$.

Intranasal steroid therapy is the mainstay of treatment for persistent AR, and it is also the preferred treatment for moderate to severe AR. Allergen immunotherapy may be indicated in AR patients who have insufficient symptom control despite medication $[4,5]$. 
However, in clinical practice, symptom control is often insufficient despite treatment, leading to a decrease in the quality of life $(\mathrm{QoL})$ of $\mathrm{AR}$ patients.

AR impairs the QoL of patients by affecting their sleep quality and their performance at work, school, or during leisure activities. AR is frequently associated with comorbidities such as asthma and conjunctivitis. Therefore, the Allergic Rhinitis and its Impact on Asthma (ARIA) guidelines have proposed criteria for AR severity, which consider the effects of AR on QoL [6]. Moreover, disease-specific QoL measurements are the best indicators of AR burden. Measuring QoL ensures that important symptoms are not overlooked and presents symptoms as an objective score, thereby allowing a comprehensive comparison of disease control status.

Stress can influence immune response, aggravate inflammation, delay wound healing, and increase susceptibility to infection [7]. Many studies have been conducted to demonstrate the correlation between stress and various allergic diseases, and it has been proven that the occurrence of allergic reactions increases when the environment is stressful [8-11]. Studies have also suggested that emotional status could affect the allergic response in various diseases [12-14]. Individuals with persistent emotional stress have been found to have frequent allergy flares [15]. However, there are limited studies on the association between stress and disease-related QoL in AR patients, and the relationship between these variables remains unclear.

We have already demonstrated in a previous study [16] involving the Allergic Rhinitis Cohort (ARCO) that stress is a risk factor for pediatric AR. In this study, it was demonstrated that children without enough play time were more likely to have AR than those with enough playtime [16]. Therefore, we hypothesized that, as observed in other chronic inflammatory diseases, perceived stress levels in AR patients might be related to diseaserelated QoL in AR patients. The purpose of this study was to evaluate the association between perceived stress and disease-related QoL in Korean adult patients with AR using the data from the ARCO study.

\section{Materials and Methods}

\subsection{Study Population and Data Collection}

This study used data from the ARCO study. The ARCO study is a national project designed to comprehensively investigate the clinical features of AR, comorbidities, and socioenvironmental factors of AR patients. It was a prospective study of Korean AR patients conducted from October 2013 to March 2015 at eight tertiary hospitals nationwide, and it was supported by the National Strategic Coordinating Center for Clinical Research under the Ministry of Health and Welfare.

Patients with AR symptoms who visited any of the eight medical centers that comprised the study sites were diagnosed with AR through history taking, endoscopic examination findings, skin prick test (SPT; Allergopharma, Reinbek, Germany) results, and serum laboratory analysis. Participants with at least one positive SPT result were considered to have AR. A total of 741 patients aged over 18 years were included in this study. The exclusion criteria were as follows: (1) patients with cardiovascular disease, serious disease involving other organs, chronic rhinosinusitis, nasal polyp, or psychiatric disorders such as depression; (2) those who had undergone sinus surgery; (3) those who could not express their opinions; and/or (4) those who could not answer the questionnaires properly.

\subsection{Survey Questionnaires}

The demographic data, AR symptom severity according to the ARIA classification (intermittent/persistent and mild/moderate-severe), perceived stress level (very low, low, moderate, or high), and disease-related QoL status on the Rhinoconjunctivitis Quality of Life Questionnaire (RQLQ) were measured using a survey questionnaire [17]. The questionnaire also included survey items on age, sex, monthly income, education, smoking status, high-risk drinking [18], number of siblings, body mass index (BMI), chronic conditions (asthma, hypertension, and diabetes), number of colds per year, global visual analogue 
scale (VAS) for AR, VAS for ocular discomfort, VAS for epiphora, perceived stress levels, and RQLQ.

The subjective degree of AR symptoms (global AR symptoms, ocular discomfort, and epiphora) was assessed using the VAS score ranging from 0 to 10 points. Perceived stress level was determined based on the response to the question $[19,20]$ "How much stress do you have in your daily life during the past 1 year", which was adopted from the Korea National Health and Nutrition Examination Survey (KNHANES) [21]. Patients were assigned scores from 0 to 3 according to their stress level: $0=$ very low, $1=$ low, $2=$ moderate, and $3=$ high. Participants were asked to grade the general stress they experienced in their daily lives, excluding that caused by AR symptoms. To measure disease-related QoL, we used the modified RQLQ from the validated Korean version, which contained 28 questions from 7 domains (activity limitations, sleep problems, nose symptoms, eye symptoms, non-nose/eye problems, practical problems, and emotional function). Participants were asked to report how bothered they were by their allergy symptoms during the previous week, and their scores ranged from 0 (not impaired at all) to 6 (severely impaired) $[17,22]$. The total RQLQ score was calculated as the mean of all 28 item scores, whereas the individual domain scores were the means of the scores of the items in those domains. Higher scores reflected worse disease-related QoL.

\subsection{Skin Prick Test and Immunological Parameters}

The SPT was performed on the medial sides of both forearms of the participants, using 13 common standardized allergen extracts (Allergopharma, Reinbek, Germany). The allergens were categorized into 7 groups: house dust mite (Dermatophagoides pteronyssinus (Dp) and Dermatophagoides farinae (Df)), animal danders (cat and dog), cockroaches (Blattella germanica), molds (Alternaria alternata and Aspergillus fumigatus), trees, grasses, and weeds (mugwort and ragweed). Histamine (1\% histamine phosphate) and $0.9 \%$ saline were used as positive and negative controls, respectively. Skin sensitization (positive SPT) was defined as an average wheal diameter in response to any allergen greater than or equal to $3 \mathrm{~mm}$. The numbers of categorized allergen groups with positive SPT results were collected. In addition, the serum total IgE levels and eosinophil counts of participants were measured via serum analysis.

\subsection{Statistical Analyses}

Patient characteristics are presented as means \pm standard deviations for continuous variables and as frequencies (proportions) for categorical variables. The relationship between perceived stress level and RQLQ score was analyzed using univariable and multiple linear regression analyses. The assumptions in the linear regression model such as linear relationship, normality, and homoscedasticity were checked by residual plots. If homoscedasticity was violated, heteroscedasticity-consistent standard error estimators (HC3) were used to estimate the standard errors of model coefficients in regression models [23]. The relationship between perceived stress and RQLQ was estimated by adjusting the known confounders and covariates using the multiple regression model. All analyses were performed using SAS version 9.4 (SAS Institute, Inc., Cary, NC, USA), and a $p$ value $<0.05$ was considered statistically significant.

\section{Results}

\subsection{General Association between Variables and RQLQ in AR Patients}

The general characteristics of the participants are presented in Table 1. The total RQLQ and score for each domain according to perceived stress level are shown in Figure 1. With univariate regression analysis, we analyzed the effect of the variables on total RQLQ (Table 2). Perceived stress levels were associated with a high total RQLQ score, which reflected poor disease-related QoL. Other variables that had a statistically significant relationship with the total RQLQ score were female sex, age, number of siblings, high-risk drinking, and number of colds per year $(p<0.05)$. As expected, the global VAS score, VAS 
score for ocular discomfort, VAS score for epiphora, persistent AR, and moderate-severe $\mathrm{AR}$, all of which are closely related to AR symptom severity, were also associated with RQLQ score. Among the laboratory findings, eosinophil counts and the number of categorized allergen groups with positive SPT results were associated with an increased total RQLQ. The level of perceived stress was associated with the total RQLQ score. The total RQLQ score was elevated by 0.592 (95\% confidence interval (CI), 0.311-0.873; $p<0.0001$ ) when the stress level was low, 1.049 (95\% CI, $0.755-1.343 ; p<0.0001)$ when the stress level was moderate, and $1.543(95 \%$ CI, $1.120-1.965 ; p=0.0001)$ when the stress level was high. Female sex increased the total RQLQ score by 0.343 (95\% CI, $0.138-0.547, p=0.0011)$.

Table 1. General characteristics of participants according to total RQLQ score and quartiles.

\begin{tabular}{|c|c|c|c|c|c|c|}
\hline Variables & & Total & Q1 & Q2 & Q3 & Q4 \\
\hline \multirow[t]{3}{*}{ RQLQ score } & $n$ & 741 & 184 & 188 & 185 & 184 \\
\hline & Mean \pm SD & $2.3 \pm 1.32$ & & & & \\
\hline & Median & 2.29 & 0.64 & 1.75 & 2.82 & 3.93 \\
\hline \multirow{4}{*}{$\begin{array}{c}\text { Perceived stress } \\
\text { level } \\
(n, \%)\end{array}$} & Very low & 87 (11.74) & $41(22.28)$ & 22 (11.7) & $15(8.11)$ & $9(4.89)$ \\
\hline & Low & $360(48.58)$ & 99 (53.8) & $101(53.72)$ & $81(43.78)$ & 79 (42.93) \\
\hline & Moderate & $250(33.74)$ & $41(22.28)$ & $58(30.85)$ & $79(42.7)$ & $72(39.13)$ \\
\hline & High & $44(5.94)$ & $3(1.63)$ & $7(3.72)$ & $10(5.41)$ & $24(13.04)$ \\
\hline \multirow{8}{*}{$\begin{array}{l}\text { Institute } \\
(n, \%)\end{array}$} & 1 & $59(7.96)$ & $17(9.24)$ & $12(6.38)$ & $18(9.73)$ & $12(6.52)$ \\
\hline & 2 & $95(12.82)$ & 31 (16.85) & $20(10.64)$ & 25 (13.51) & 19 (10.33) \\
\hline & 3 & $125(16.87)$ & 25 (13.59) & 34 (18.09) & $31(16.76)$ & 35 (19.02) \\
\hline & 4 & $59(7.96)$ & $19(10.33)$ & $22(11.7)$ & $9(4.86)$ & $9(4.89)$ \\
\hline & 5 & 121 (16.33) & $15(8.15)$ & 37 (19.68) & 33 (17.84) & 36 (19.57) \\
\hline & 6 & 95 (12.82) & $24(13.04)$ & $23(12.23)$ & $23(12.43)$ & 25 (13.59) \\
\hline & 7 & 90 (12.15) & 34 (18.48) & $17(9.04)$ & $20(10.81)$ & 19 (10.33) \\
\hline & 8 & 97 (13.09) & $19(10.33)$ & $23(12.23)$ & $26(14.05)$ & $29(15.76)$ \\
\hline Age & Mean \pm SD & $32.36 \pm 12.72$ & $34.78 \pm 13.98$ & $31.79 \pm 13.27$ & $31.64 \pm 11.81$ & $31.25 \pm 11.45$ \\
\hline \multirow{2}{*}{$\begin{array}{l}\text { Sex } \\
(n, \%)\end{array}$} & Female & $242(32.66)$ & $51(27.72)$ & $48(25.53)$ & $69(37.3)$ & $74(40.22)$ \\
\hline & Male & 499 (67.34) & 133 (72.28) & 140 (74.47) & $116(62.7)$ & $110(59.78)$ \\
\hline \multirow{4}{*}{$\begin{array}{c}\text { Income } \\
\text { (Korean won, } \\
\text { KRW) }\end{array}$} & $<2 \mathrm{M}$ & 94 (12.69) & $20(10.87)$ & $22(11.7)$ & $26(14.05)$ & $26(14.13)$ \\
\hline & $2 \mathrm{M}-4 \mathrm{M}$ & $236(31.85)$ & $59(32.07)$ & 57 (30.32) & $63(34.05)$ & 57 (30.98) \\
\hline & $4 \mathrm{M}-6 \mathrm{M}$ & 187 (25.24) & 49 (26.63) & 45 (23.94) & $51(27.57)$ & $42(22.83)$ \\
\hline & $>6 \mathrm{M}$ & $224(30.23)$ & $56(30.43)$ & $64(34.04)$ & $45(24.32)$ & 59 (32.07) \\
\hline \multirow[t]{5}{*}{ Education } & 1 (lowest) & $11(1.48)$ & $4(2.17)$ & $4(2.13)$ & $1(0.54)$ & $2(1.09)$ \\
\hline & 2 & $38(5.13)$ & $9(4.89)$ & $10(5.32)$ & $12(6.49)$ & $7(3.8)$ \\
\hline & 3 & $336(45.34)$ & 95 (51.63) & 83 (44.15) & $74(40)$ & 84 (45.65) \\
\hline & 4 & 304 (41.03) & $62(33.7)$ & $69(36.7)$ & $90(48.65)$ & $83(45.11)$ \\
\hline & 5 & $52(7.02)$ & $14(7.61)$ & $22(11.7)$ & $8(4.32)$ & $8(4.35)$ \\
\hline $\begin{array}{l}\text { Number of } \\
\text { siblings }\end{array}$ & Mean \pm SD & $1.89 \pm 1.61$ & $2.14 \pm 1.76$ & $1.86 \pm 1.73$ & $1.74 \pm 1.42$ & $1.83 \pm 1.49$ \\
\hline $\operatorname{BMI}\left(\mathrm{kg} / \mathrm{m}^{2}\right)$ & Mean \pm SD & $23.34 \pm 3.46$ & $23.56 \pm 3.44$ & $23.35 \pm 3.75$ & $23.18 \pm 3.36$ & $23.27 \pm 3.29$ \\
\hline \multirow[t]{2}{*}{$\begin{array}{l}\text { High-risk } \\
\text { drinking }\end{array}$} & No & 711 (95.95) & 178 (96.74) & $184(97.87)$ & $181(97.84)$ & 168 (91.3) \\
\hline & Yes & $30(4.05)$ & $6(3.26)$ & $4(2.13)$ & $4(2.16)$ & $16(8.7)$ \\
\hline \multirow[t]{3}{*}{ Smoking status } & Non-smoker & $435(58.7)$ & 107 (58.15) & $116(61.7)$ & $111(60)$ & $101(54.89)$ \\
\hline & Ex-smoker & $142(19.16)$ & $43(23.37)$ & $32(17.02)$ & $28(15.14)$ & $39(21.2)$ \\
\hline & Smoker & $164(22.13)$ & 34 (18.48) & $40(21.28)$ & $46(24.86)$ & $44(23.91)$ \\
\hline \multirow[t]{2}{*}{ Asthma } & No & 669 (90.28) & $166(90.22)$ & 172 (91.49) & 165 (89.19) & $166(90.22)$ \\
\hline & Yes & $72(9.72)$ & $18(9.78)$ & $16(8.51)$ & $20(10.81)$ & $18(9.78)$ \\
\hline
\end{tabular}


Table 1. Cont.

\begin{tabular}{|c|c|c|c|c|c|c|}
\hline Variables & & Total & Q1 & Q2 & Q3 & Q4 \\
\hline \multirow[t]{2}{*}{ Hypertension } & No & 699 (94.33) & $169(91.85)$ & $175(93.09)$ & $181(97.84)$ & $174(94.57)$ \\
\hline & Yes & $42(5.67)$ & 15 (8.15) & $13(6.91)$ & $4(2.16)$ & $10(5.43)$ \\
\hline \multirow[t]{2}{*}{ Diabetes } & No & 721 (97.3) & $178(96.74)$ & $179(95.21)$ & $183(98.92)$ & 181 (98.37) \\
\hline & Yes & $20(2.7)$ & $6(3.26)$ & $9(4.79)$ & $2(1.08)$ & $3(1.63)$ \\
\hline $\begin{array}{l}\text { Number of } \\
\text { colds per year }\end{array}$ & Mean \pm SD & $2.87 \pm 3.12$ & $2.22 \pm 2.24$ & $3.03 \pm 3.77$ & $3.14 \pm 3.14$ & $3.1 \pm 3.05$ \\
\hline $\begin{array}{c}\text { Global VAS } \\
\text { score }\end{array}$ & Mean \pm SD & $6.84 \pm 1.95$ & $5.48 \pm 2.14$ & $6.51 \pm 1.72$ & $7.37 \pm 1.37$ & $8.03 \pm 1.52$ \\
\hline $\begin{array}{l}\text { VAS score for } \\
\text { eye discomfort }\end{array}$ & Mean \pm SD & $3.94 \pm 3.22$ & $1.99 \pm 2.57$ & $3.19 \pm 2.93$ & $4.52 \pm 3.12$ & $6.05 \pm 2.75$ \\
\hline $\begin{array}{l}\text { VAS score for } \\
\text { epiphora }\end{array}$ & Mean \pm SD & $2.08 \pm 2.73$ & $0.69 \pm 1.3$ & $1.32 \pm 2.04$ & $2.49 \pm 2.84$ & $3.83 \pm 3.2$ \\
\hline \multirow[t]{2}{*}{$\begin{array}{c}\text { ARIA } \\
\text { classification of } \\
\text { AR }\end{array}$} & Intermittent AR & $295(39.81)$ & 117 (63.59) & $80(42.55)$ & $57(30.81)$ & $41(22.28)$ \\
\hline & Persistent AR & $446(60.19)$ & $67(36.41)$ & $108(57.45)$ & $128(69.19)$ & $143(77.72)$ \\
\hline \multirow[t]{2}{*}{$\begin{array}{c}\text { ARIA } \\
\text { classification of } \\
\text { AR }\end{array}$} & Mild & $101(13.63)$ & $76(41.3)$ & $19(10.11)$ & $5(2.7)$ & $1(0.54)$ \\
\hline & $\begin{array}{l}\text { Moderate- } \\
\text { severe }\end{array}$ & $640(86.37)$ & $108(58.7)$ & $169(89.89)$ & $180(97.3)$ & $183(99.46)$ \\
\hline \multirow{2}{*}{$\begin{array}{l}\text { Eosinophil } \\
\text { count } \\
\text { (cells } / \mu \mathrm{L} \text { ) }\end{array}$} & $n$ & 662 & 159 & 170 & 167 & 166 \\
\hline & Mean \pm SD & $266.84 \pm 195.45$ & $247.58 \pm 184.18$ & $243.59 \pm 150.46$ & $259.93 \pm 193.02$ & $316.05 \pm 237.67$ \\
\hline $\begin{array}{l}\text { Total IgE } \\
(\mathrm{kU} / \mathrm{L})\end{array}$ & $\begin{array}{c}n \\
\text { Mean }+ \text { SD }\end{array}$ & $\begin{array}{c}517 \\
31575+428.29\end{array}$ & $\begin{array}{c}135 \\
32589+42132\end{array}$ & $\begin{array}{c}130 \\
28719+40815\end{array}$ & $\begin{array}{c}126 \\
30454+40586\end{array}$ & $\begin{array}{c}126 \\
345.57+47778\end{array}$ \\
\hline $\begin{array}{c}\text { Number of } \\
\text { positive SPT } \\
\text { allergen groups }\end{array}$ & $\begin{array}{c}n \\
\text { Mean } \pm \text { SD }\end{array}$ & $\begin{array}{c}693 \\
2.59 \pm 1.56\end{array}$ & $\begin{array}{c}171 \\
2.3 \pm 1.38\end{array}$ & $\begin{array}{c}176 \\
2.56 \pm 1.54\end{array}$ & $\begin{array}{c}173 \\
2.65 \pm 1.7\end{array}$ & $\begin{array}{c}173 \\
2.84 \pm 1.58\end{array}$ \\
\hline
\end{tabular}

AR: allergic rhinitis; ARIA: Allergic Rhinitis and its Impact on Asthma; BMI: body mass index; M: million; Q: quartile; RQLQ: rhinoconjunctivitis quality of life questionnaire; SPT: skin prick test; VAS: visual analogue scale.

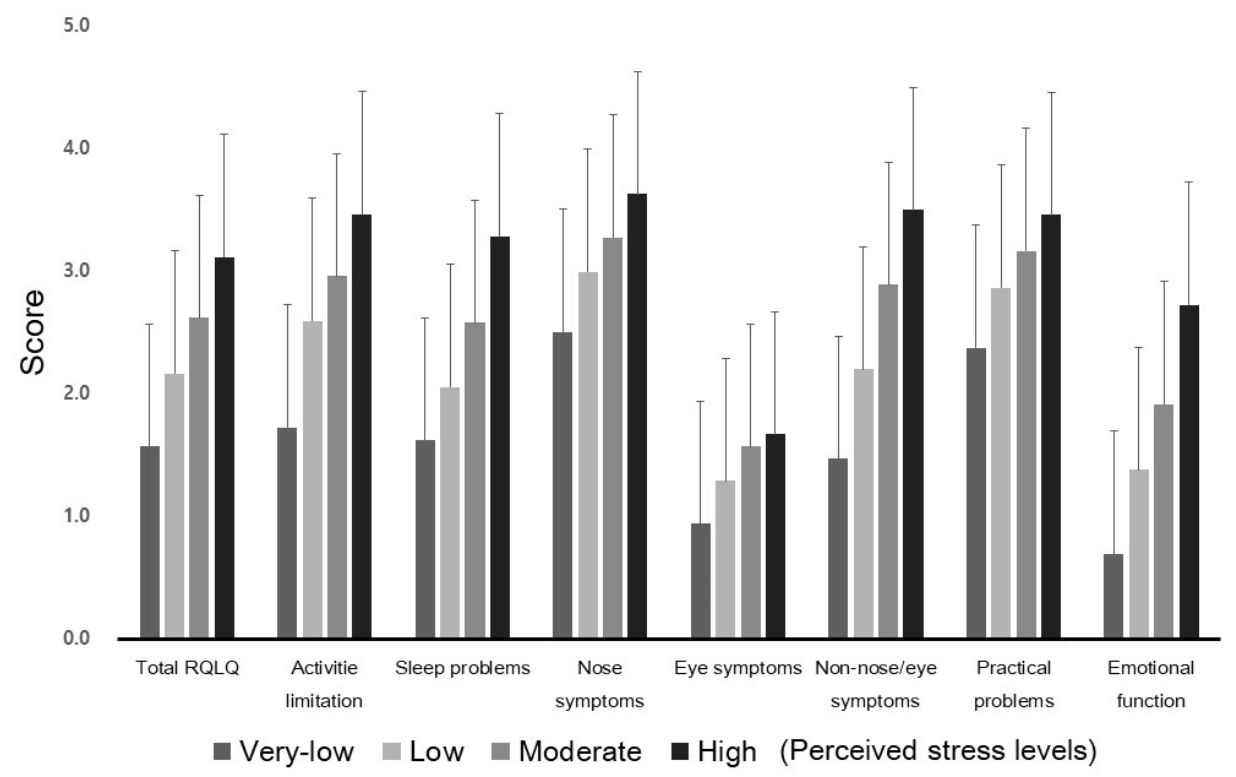

Figure 1. Total RQLQ score and domain scores according to perceived stress levels. The scores are shown as means with standard deviation. RQLQ: rhinoconjunctivitis quality of life questionnaire. 
Table 2. Univariable regressions of the variables of the patients with AR for disease-related quality of life score (RQLQ score).

\begin{tabular}{|c|c|c|c|c|c|c|}
\hline \multirow{5}{*}{$\begin{array}{l} \\
\text { Perceived stress } \\
\text { level } \\
\text { (reference = very } \\
\text { low) }\end{array}$} & \multirow[b]{3}{*}{ Low } & \multirow{3}{*}{$\begin{array}{c}\text { Estimate } \\
0.592\end{array}$} & \multicolumn{4}{|c|}{ HCSE Estimator } \\
\hline & & & \multirow{2}{*}{$\frac{\text { SE }}{0.143}$} & \multicolumn{2}{|c|}{ 95\% Confidence Interval } & \multirow{2}{*}{$\begin{array}{l}p \text {-Value } \\
<0.0001 *\end{array}$} \\
\hline & & & & 0.311 & 0.873 & \\
\hline & Moderate & 1.049 & 0.150 & 0.755 & 1.343 & $<0.0001 *$ \\
\hline & High & 1.543 & 0.215 & 1.120 & 1.965 & $<0.0001$ * \\
\hline \multirow{7}{*}{$\begin{array}{c}\text { Institute } \\
(\text { reference }=1)\end{array}$} & 2 & -0.115 & 0.220 & -0.547 & 0.317 & 0.6013 \\
\hline & 3 & 0.195 & 0.208 & -0.213 & 0.604 & 0.3484 \\
\hline & 4 & -0.329 & 0.241 & -0.803 & 0.144 & 0.1724 \\
\hline & 5 & 0.446 & 0.203 & 0.048 & 0.844 & 0.0281 \\
\hline & 6 & 0.080 & 0.223 & -0.359 & 0.518 & 0.7214 \\
\hline & 7 & -0.160 & 0.229 & -0.611 & 0.290 & 0.4851 \\
\hline & 8 & 0.333 & 0.221 & -0.101 & 0.768 & 0.1322 \\
\hline Age & & -0.009 & 0.004 & -0.017 & -0.001 & 0.0213 * \\
\hline Female & & 0.343 & 0.104 & 0.138 & 0.547 & $0.0011 *$ \\
\hline \multirow{3}{*}{$\begin{array}{c}\text { Income } \\
\text { (reference }=\text { less } \\
\text { than } 2 \mathrm{M} \\
\text { Korean won) }\end{array}$} & $2 \mathrm{M}-4 \mathrm{M}$ & -0.173 & 0.162 & -0.492 & 0.146 & 0.2877 \\
\hline & $4 \mathrm{M}-6 \mathrm{M}$ & -0.218 & 0.165 & -0.541 & 0.106 & 0.1871 \\
\hline & $>6 \mathrm{M}$ & -0.269 & 0.161 & -0.585 & 0.047 & 0.095 \\
\hline \multirow{4}{*}{$\begin{array}{l}\text { Education } \\
\text { (reference = 1, } \\
\text { lowest) }\end{array}$} & 2 & 0.237 & 0.447 & -0.640 & 1.114 & 0.5955 \\
\hline & 3 & 0.238 & 0.401 & -0.550 & 1.025 & 0.5535 \\
\hline & 4 & 0.476 & 0.402 & -0.312 & 1.265 & 0.236 \\
\hline & 5 & -0.071 & 0.431 & -0.916 & 0.775 & 0.8698 \\
\hline $\begin{array}{l}\text { Number of } \\
\text { siblings }\end{array}$ & & -0.065 & 0.031 & -0.126 & -0.004 & $0.0367 *$ \\
\hline BMI & & -0.012 & 0.013 & -0.038 & 0.015 & 0.3817 \\
\hline $\begin{array}{c}\text { High-risk } \\
\text { drinking }\end{array}$ & & 0.632 & 0.280 & 0.083 & 1.181 & $0.0241 *$ \\
\hline \multirow{2}{*}{$\begin{array}{l}\text { Smoking status } \\
\text { (reference }=\text { non- } \\
\text { smoker) }\end{array}$} & Ex-smoker & -0.013 & 0.139 & -0.287 & 0.261 & 0.9267 \\
\hline & Smoker & 0.098 & 0.115 & -0.127 & 0.324 & 0.3918 \\
\hline Asthma & & 0.046 & 0.161 & -0.269 & 0.361 & 0.7747 \\
\hline Hypertension & & -0.309 & 0.230 & -0.760 & 0.142 & 0.1791 \\
\hline Diabetes & & -0.395 & 0.347 & -1.077 & 0.287 & 0.2555 \\
\hline $\begin{array}{l}\text { Number of } \\
\text { colds per year }\end{array}$ & & 0.046 & 0.016 & 0.016 & 0.077 & $0.003 *$ \\
\hline $\begin{array}{c}\text { Global VAS } \\
\text { score }\end{array}$ & & 0.337 & 0.021 & 0.295 & 0.379 & $<0.0001$ * \\
\hline $\begin{array}{l}\text { VAS score for } \\
\text { eye discomfort }\end{array}$ & & 0.197 & 0.013 & 0.170 & 0.223 & $<0.0001^{*}$ \\
\hline $\begin{array}{l}\text { VAS score for } \\
\text { epiphora }\end{array}$ & & 0.224 & 0.015 & 0.195 & 0.253 & $<0.0001$ * \\
\hline $\begin{array}{c}\text { ARIA } \\
\text { classification of } \\
\text { AR, persistent }\end{array}$ & & 0.865 & 0.093 & 0.682 & 1.047 & $<0.0001$ * \\
\hline
\end{tabular}


Table 2. Cont.

\begin{tabular}{|c|c|c|c|c|c|}
\hline & \multirow[b]{2}{*}{ Estimate } & \multicolumn{4}{|c|}{ HCSE Estimator } \\
\hline & & SE & $95 \%$ Cor & nterval & $p$-Value \\
\hline $\begin{array}{l}\text { classification of } \\
\text { AR, moderate- } \\
\text { severe }\end{array}$ & 1.673 & 0.090 & 1.496 & 1.850 & $<0.0001$ * \\
\hline $\begin{array}{l}\text { Eosinophil } \\
\text { count }\end{array}$ & 0.001 & 0.0003 & 0.0005 & 0.002 & $0.0002 *$ \\
\hline Total IgE & 0.0001 & 0.0001 & -0.0002 & 0.0004 & 0.5199 \\
\hline $\begin{array}{l}\text { Number of } \\
\text { positive SPT } \\
\text { allergen groups }\end{array}$ & 0.112 & 0.031 & 0.052 & 0.172 & $0.0003 *$ \\
\hline
\end{tabular}

AR: allergic rhinitis; ARIA: Allergic Rhinitis and its Impact on Asthma; BMI: body mass index; M: million; RQLQ: rhinoconjunctivitis quality of life questionnaire; SPT: skin prick test; VAS: visual analogue scale. ${ }^{*}$ Univariate regression analyses, significance at $p<0.05$. Heteroscedasticity-consistent standard error estimators, HC3, were used.

\subsection{Association between Perceived Stress Levels and Total RQLQ}

To analyze the effect of stress levels on total RQLQ score, the multiple regression model was adjusted for institute, age, sex, income, education, smoking status, high-risk drinking, number of siblings, BMI, asthma, hypertension, diabetes, number of colds per year, global VAS score, VAS score for ocular discomfort, VAS score for epiphora, AR severity based on ARIA classification, eosinophil count, total IgE, and number of positive allergen groups on the SPT (Table 3). Perceived stress level was associated with the total RQLQ score (Table 3). A low stress level increased the total RQLQ by 0.353 (reference, very-low-level-stress group; 95\% CI, 0.099-0.606; $p=0.0065)$. The moderate and high stress levels increased the total RQLQ by 0.597 (95\% CI, $0.327-0.867 ; p<0.0001)$ and 1.210 (95\% CI, $0.831-1.589 ; p<0.0001$ ), respectively.

\subsection{Association between Perceived Stress Levels and Individual RQLQ Domain Scores}

In the analyses of individual RQLQ domain scores, all domains except for practical problems (inconvenience of having to carry tissues, the need to rub the nose or eye, and the need to blow the nose repeatedly) and eye symptoms had significantly high RQLQ scores, especially in patients with a high level of perceived stress (Table 3). The domains for sleep problems and nasal symptoms were affected in the high-level-of-perceived-stress group compared with the very-low-level-stress group. The domains for activity limitations, non-nose/eye problems, and emotional function were affected in all the levels of the perceived stress group compared with the control group. The emotional function (2.081, 95\% CI; 1.378-2.783; $p<0.0001)$ and activity limitation $(1.633,95 \%$ CI; $0.906-2.360 ; p<$ $0.0001)$ domains were most impaired, followed by the non-nose/eye problem domain $(1.619,95 \%$ CI; $1.015-2.223 ; p<0.0001)$ and sleep disturbance domain $(1.412 ; 95 \%$ CI; $0.667-2.157 ; p=0.0002)$ in the high-perceived-stress group. The nasal symptom domain score showed a significant increment in the high perceived stress group $(0.717 ; 95 \% \mathrm{CI}$; 0.083-1.350; $p=0.0267)$. 
Table 3. The adjusted association between perceived stress level and RQLQ score.

\begin{tabular}{|c|c|c|c|c|c|c|}
\hline \multirow{5}{*}{$\begin{array}{c}\text { RQLQ Domain } \\
\text { Total }\end{array}$} & \multirow{5}{*}{$\begin{array}{c}\text { Perceived } \\
\text { Stress Level } \\
\text { Low } \\
\text { Moderate } \\
\text { High }\end{array}$} & \multirow{3}{*}{$\begin{array}{c}\text { Estimate } \\
0.353\end{array}$} & \multicolumn{4}{|c|}{ HCSE Estimator } \\
\hline & & & \multirow{2}{*}{$\begin{array}{c}\text { SE } \\
0.129\end{array}$} & \multicolumn{2}{|c|}{ 95\% Confidence Interval } & \multirow{2}{*}{$\frac{p \text {-Value }}{0.0065 \text { * }}$} \\
\hline & & & & 0.099 & 0.606 & \\
\hline & & 0.597 & 0.138 & 0.327 & 0.867 & $<0.0001$ * \\
\hline & & 1.210 & 0.193 & 0.831 & 1.589 & $<0.0001$ * \\
\hline \multirow[t]{3}{*}{ Activity limitations } & Low & 0.700 & 0.242 & 0.225 & 1.175 & $0.0039 *$ \\
\hline & Moderate & 0.920 & 0.256 & 0.418 & 1.422 & $0.0004 *$ \\
\hline & High & 1.633 & 0.370 & 0.906 & 2.360 & $<0.0001$ * \\
\hline \multirow[t]{3}{*}{ Sleep problems } & Low & 0.025 & 0.250 & -0.466 & 0.515 & 0.9217 \\
\hline & Moderate & 0.411 & 0.263 & -0.106 & 0.927 & 0.119 \\
\hline & High & 1.412 & 0.379 & 0.667 & 2.157 & $0.0002 *$ \\
\hline \multirow[t]{3}{*}{ Nasal symptoms } & Low & 0.369 & 0.213 & -0.050 & 0.788 & 0.0843 \\
\hline & Moderate & 0.424 & 0.220 & -0.009 & 0.857 & 0.0552 \\
\hline & High & 0.717 & 0.322 & 0.083 & 1.350 & $0.0267 *$ \\
\hline \multirow[t]{3}{*}{ Eye symptoms } & Low & 0.020 & 0.137 & -0.250 & 0.290 & 0.8869 \\
\hline & Moderate & 0.019 & 0.148 & -0.271 & 0.310 & 0.8967 \\
\hline & High & 0.190 & 0.220 & -0.243 & 0.623 & 0.3883 \\
\hline \multirow[t]{3}{*}{$\begin{array}{l}\text { Non-nose/eye } \\
\text { symptoms }\end{array}$} & Low & 0.434 & 0.168 & 0.104 & 0.763 & $0.01 *$ \\
\hline & Moderate & 0.846 & 0.179 & 0.494 & 1.198 & $<0.0001 *$ \\
\hline & High & 1.619 & 0.307 & 1.015 & 2.223 & $<0.0001$ * \\
\hline \multirow[t]{3}{*}{ Practical problems } & Low & 0.309 & 0.218 & -0.119 & 0.737 & 0.157 \\
\hline & Moderate & 0.363 & 0.229 & -0.088 & 0.813 & 0.1141 \\
\hline & High & 0.487 & 0.360 & -0.220 & 1.194 & 0.1768 \\
\hline \multirow[t]{3}{*}{ Emotional function } & Low & 0.546 & 0.156 & 0.241 & 0.852 & 0.0005 * \\
\hline & Moderate & 0.984 & 0.174 & 0.643 & 1.326 & $<0.0001 *$ \\
\hline & High & 2.081 & 0.357 & 1.378 & 2.783 & $<0.0001$ * \\
\hline
\end{tabular}

RQLQ: rhinoconjunctivitis quality of life questionnaire. Multiple linear regression with heteroscedasticity-consistent standard errors (HSCE) for RQLQ score $\left(n=467\right.$, reference $=$ very-low-level-stress group). ${ }^{*}$ Linear regression analyses, significance at $p<0.05$. The adjusted covariates in the model were institute, age, sex, income, education, smoking status, high-risk drinking, number of siblings, BMI, asthma, hypertension, diabetes, number of colds per year, global VAS, VAS for ocular discomfort, VAS for epiphora, AR severity based on ARIA classification, eosinophil count, total IgE, and positive allergen group number on SPT.

\subsection{Contribution of Perceived Stress Levels to Total RQLQ}

The adjusted $\mathrm{R}^{2}$ for the total RQLQ scores in AR participants was 0.5279 , and the perceived stress levels contributed $4.08 \%$ in additional explanatory power to the total RQLQ score in AR patients (Table 4). The association between perceived stress level and total RQLQ score could be dependent on AR severity. Therefore, we additionally analyzed the interactions between AR severity and the total RQLQ; however, the relationship was not significant $(p=0.8176)$.

Table 4. Crude and adjusted $R^{2}$ for RQLQ scores in AR participants.

\begin{tabular}{lcc}
\hline & R-Square & Adjusted $\mathbf{R}^{2 *}$ \\
\hline Without perceived stress level & 0.5234 & 0.4871 \\
With perceived stress level & 0.5644 & 0.5279 \\
\hline
\end{tabular}

AR: allergic rhinitis; RQLQ: rhinoconjunctivitis quality of life questionnaire. * Stratified model with sociodemographics, chronic conditions, AR severity (according to ARIA classification and VAS), laboratory data (eosinophil counts, skin prick test results, and total IgE) and perceived stress level (very low, low, moderate, and high).

\section{Discussion}

In this study, stress level, which was easily indexed by simple questions, was significantly correlated with RQLQ score in AR patients. AR patients with a high level 
of perceived stress showed a lower disease-related QoL than those with a low level of perceived stress. These findings were consistent regardless of AR severity. To the best of our knowledge, this is the first report on the negative effect of perceived stress on disease-related QoL in AR patients.

The minimal clinically important difference of the RQLQ score has been found to be 0.5 on a scale ranging from 0 to 6 for the total RQLQ score [24]. In the current study, we demonstrated that perceived stress was associated with a significant elevation in RQLQ score of 0.592, even if the perceived stress level was low. Furthermore, a high perceived stress level was associated with a significant increase in the RQLQ score of 1.543.

There are very few previous studies on the relationship between stress and QoL in AR patients. In one study, $73.5 \%$ of patients with persistent AR had a positive result on the Kessler Psychological Distress Scale, which indicated a close association between persistent AR and psychological stress [15]. Patients with persistent AR who had a positive result on the Kessler Psychological Distress Scale and who were managed with antihistamines with imipramine demonstrated an improved QoL compared with the AR patients who were treated without imipramine. Kimata et al. [25] reported that stress-relieving activities such as listening to soothing music might decrease allergic responses by decreasing allergenspecific IgE levels. In our study, the multiple regression model adjusted for AR-related variables showed that $4.08 \%$ of the RQLQ score was explained by perceived stress.

We clearly demonstrated the effect of perceived stress on disease-related QoL in AR patients by classifying their perceived stress levels into four categories, using a simple question. We assessed the participants' perceived stress levels based on their responses on a one-item questionnaire without using comprehensive stress measuring methods such as the Perceived Stress Scale (PSS) or the Daily Hassles Scale (DHS) [26]. This method of stratifying the stress level was simple and easy, and it enabled us to adequately assess the stress levels of AR patients with minimal effort. However, our use of such a simple categorization method might have resulted in the misclassification of the levels of perceived stress in the current study, although there was some evidence that the question we used had sufficient reliability for assessing stress levels [27].

There is accumulating evidence linking psychological stress to the expression of atopic disorders such as AR. Stress might have a direct effect and an important role in the onset and exacerbation of atopic disease through neuroendocrine and immunologic alterations [28-31]. Stress has been strongly associated with asthma incidence and hospitalization, use of asthma medication, AR, and atopic dermatitis in adults [29]. Moreover, there have been reports that maternal parenting and prenatal stress are related to the incidence of allergic diseases in children [32,33], suggesting the need for a multifaceted understanding of the relationship between stress and allergic diseases. As shown in our study, perceived stress was significantly associated with decreased disease-related QoL (high RQLQ score). Further study is needed to elucidate whether stress management leads to the improvement of QoL in AR patients. Studies exploring interventions such as medications, behavioral treatment, mental support, lifestyle modification, and adjuvant methods for relieving stress in the daily lives in AR patients receiving adequate medical treatment for allergic symptoms can further clarify the effect of stress on AR treatment.

We analyzed the total RQLQ score, which was the average of the sum of the scores of each item in seven domains and explored the possibility that a particular RQLQ domain might be more affected by perceived stress than others. Perceived stress led to impairments in both overall QoL as well as almost all the domains of rhinitis-related QoL such as nasal symptoms, non-nose/eye problems, sleep problems, activity limitations, and emotional function. Given that each domain reflected rhinitis symptom-related problems and the results were adjusted for AR severity, it was clear that perceived stress had an overall significant impact on rhinitis-related QoL. In addition to the medical and surgical treatment of allergic rhinitis, perceived stress in AR patients should be adequately assessed and actively managed to improve disease-related QoL. 
The perceived stress of AR patients should be assessed in a feasible way at the clinic or bedside, and stress management should be considered as one of the strategies for managing AR. Based on the results of this study, a systematic approach for assessing and managing stress should be considered to be an important factor for improving the QoL of AR patients.

Although it provides evidence for the association between a high stress level and poor, disease-related QoL in AR patients, our study nonetheless has several limitations. First, while the simple question used to evaluate perceived stress in this study was very useful, further evaluation using various measures for evaluating stress might be more useful to study the association between stress and AR-related QoL. Second, as this was a cross-sectional study, the temporal link between perceived stress and disease-related QoL could not be assessed. Third, as we used self-reported data gathered using a questionnaire, there was a risk of imprecise recall and misclassification of perceived stress and QoL status.

\section{Conclusions}

AR patients with a high level of perceived stress showed a lower disease-related QoL compared with those with a low level of perceived stress. These findings were observed regardless of AR severity. These results highlight the need for clinicians to be aware of the stress status of AR patients. Controlling rhinitis symptoms is also important for improving the disease-related QoL of AR patients. Moreover, our results emphasize the importance of stress as a modifiable determinant of disease-related QoL. Assessment and active management of stress in AR patients may improve their disease-related QoL.

Author Contributions: Conceptualization, I.G.K. and D.H.H.; methodology, H.Y., M.J.K., and Y.C.; writing-original draft preparation, I.G.K., J.W.L., and M.J.K.; writing-review and editing, I.G.K. and D.H.H.; supervision, C.-S.R., Y.C., and D.H.H.; funding acquisition, C.-S.R. and D.H.H. All authors have read and agreed to the published version of the manuscript.

Funding: The ARCO study was conducted with the support of the National Strategic Coordinating Center for Clinical Research, Ministry of Health and Welfare (Grant No. HI10C2020). The electronic CRF development and data management for this study were performed using the internet-based Clinical Research and Trial management system (iCReaT), a data management system established by the Centers for Disease Control and Prevention, Ministry of Health and Welfare (iCReaT Study No. C140003).

Institutional Review Board Statement: The ARCO study was registered at the Clinical Research Information Service, Korea Centers for Disease Control and Prevention, Ministry of Health and Welfare (Republic of Korea), 2010 (KCT0000997, https: / bit.ly/2SbU1gn, accessed on 16 August 2021). The study was conducted according to the guidelines of the Declaration of Helsinki and approved by the Institutional Review Board of each hospital. The ethics committee of Seoul National University approved the study protocol and the informed consent statements (Approval No. B1308/216-003).

Informed Consent Statement: Informed consent was obtained from all participants involved in the study.

Data Availability Statement: All data are available upon request.

Acknowledgments: The authors thank all the members of the ARCO Study Group for their contribution to the data collection through patient recruiting: Jung Soo Kim and Sung Jae Heo, Kyungpook National University Hospital; Kyu-Sup Cho, Pusan National University Hospital; Hyo Yeol Kim, Samsung Medical Center; Young Hyo Kim, Inha University Hospital; Sam Hyun Kwon and Jong Seung Kim, Jeonbuk National University Hospital; Yong Min Kim, Chungnam National University Hospital.

Conflicts of Interest: The authors declare no conflict of interest. The funders had no role in the design of the study; in the collection, analyses, or interpretation of data; in the writing of the manuscript; or in the decision to publish the results. 


\section{References}

1. Asher, M.I.; Montefort, S.; Bjorksten, B.; Lai, C.K.; Strachan, D.P.; Weiland, S.K.; Williams, H.; Group, I.P.T.S. Worldwide time trends in the prevalence of symptoms of asthma, allergic rhinoconjunctivitis, and eczema in childhood: ISAAC Phases One and Three repeat multicountry cross-sectional surveys. Lancet 2006, 368, 733-743. [CrossRef]

2. Ait-Khaled, N.; Pearce, N.; Anderson, H.R.; Ellwood, P.; Montefort, S.; Shah, J.; ISAAC Phase Three Study Group. Global map of the prevalence of symptoms of rhinoconjunctivitis in children: The International Study of Asthma and Allergies in Childhood (ISAAC) Phase Three. Allergy 2009, 64, 123-148. [CrossRef]

3. Rhee, C.S.; Wee, J.H.; Ahn, J.C.; Lee, W.H.; Tan, K.L.; Ahn, S.; Lee, J.H.; Lee, C.H.; Cho, Y.S.; Park, K.H.; et al. Prevalence, risk factors and comorbidities of allergic rhinitis in South Korea: The Fifth Korea National Health and Nutrition Examination Survey. Am. J. Rhinol. Allergy 2014, 28, e107-e114. [CrossRef]

4. Brozek, J.L.; Bousquet, J.; Agache, I.; Agarwal, A.; Bachert, C.; Bosnic-Anticevich, S.; Brignardello-Petersen, R.; Canonica, G.W.; Casale, T.; Chavannes, N.H.; et al. Allergic Rhinitis and its Impact on Asthma (ARIA) guidelines-2016 revision. J. Allergy Clin. Immunol. 2017, 140, 950-958. [CrossRef]

5. Seidman, M.D.; Gurgel, R.K.; Lin, S.Y.; Schwartz, S.R.; Baroody, F.M.; Bonner, J.R.; Dawson, D.E.; Dykewicz, M.S.; Hackell, J.M.; Han, J.K.; et al. Clinical practice guideline: Allergic rhinitis executive summary. Otolaryngol. Head Neck Surg. 2015, 152, 197-206. [CrossRef]

6. Bousquet, J.; Khaltaev, N.; Cruz, A.A.; Denburg, J.; Fokkens, W.J.; Togias, A.; Zuberbier, T.; Baena-Cagnani, C.E.; Canonica, G.W.; van Weel, C.; et al. Allergic Rhinitis and its Impact on Asthma (ARIA) 2008 update (in collaboration with the World Health Organization, GA(2)LEN and AllerGen). Allergy 2008, 63 (Suppl. 86), 8-160. [CrossRef]

7. Glaser, R.; Kiecolt-Glaser, J.K. Stress-induced immune dysfunction: Implications for health. Nat. Rev. Immunol. 2005, 5, 243-251. [CrossRef]

8. Liu, L.Y.; Coe, C.L.; Swenson, C.A.; Kelly, E.A.; Kita, H.; Busse, W.W. School examinations enhance airway inflammation to antigen challenge. Am. J. Respir. Crit. Care Med. 2002, 165, 1062-1067. [CrossRef] [PubMed]

9. Schmid-Ott, G.; Jaeger, B.; Adamek, C.; Koch, H.; Lamprecht, F.; Kapp, A.; Werfel, T. Levels of circulating CD8(+) T lymphocytes, natural killer cells, and eosinophils increase upon acute psychosocial stress in patients with atopic dermatitis. J. Allergy Clin. Immunol. 2001, 107, 171-177. [CrossRef] [PubMed]

10. Kimata, H. Enhancement of allergic skin wheal responses in patients with atopic eczema/dermatitis syndrome by playing video games or by a frequently ringing mobile phone. Eur. J. Clin. Investig. 2003, 33, 513-517. [CrossRef] [PubMed]

11. Heffner, K.L.; Kiecolt-Glaser, J.K.; Glaser, R.; Malarkey, W.B.; Marshall, G.D. Stress and anxiety effects on positive skin test responses in young adults with allergic rhinitis. Ann. Allergy Asthma Immunol. 2014, 113, 13-18. [CrossRef] [PubMed]

12. Kaye, J.; Morton, J.; Bowcutt, M.; Maupin, D. Stress, depression, and psychoneuroimmunology. J. Neurosci. Nurs. 2000, 32, 93-100. [CrossRef] [PubMed]

13. Kimata, H. Emotion with tears decreases allergic responses to latex in atopic eczema patients with latex allergy. J. Psychosom. Res. 2006, 61, 67-69. [CrossRef] [PubMed]

14. Koch, A.K.; Schols, M.; Langhorst, J.; Dobos, G.; Cramer, H. Perceived stress mediates the effect of yoga on quality of life and disease activity in ulcerative colitis. Secondary analysis of a randomized controlled trial. J. Psychosom. Res. 2020, $130,109917$. [CrossRef] [PubMed]

15. El Hennawi Del, D.; Ahmed, M.R.; Farid, A.M. Psychological stress and its relationship with persistent allergic rhinitis. Eur. Arch. Otorhinolaryngol. 2016, 273, 899-904. [CrossRef]

16. Han, D.H.; Ahn, J.C.; Mun, S.J.; Park, S.K.; Oh, S.Y.; Rhee, C.S. Novel Risk Factors for Allergic Rhinitis in Korean Elementary School Children: ARCO-kids Phase II in a Community. Allergy Asthma Immunol. Res. 2015, 7, 234-240. [CrossRef]

17. Juniper, E.F.; Guyatt, G.H. Development and testing of a new measure of health status for clinical trials in rhinoconjunctivitis. Clin. Exp. Allergy 1991, 21,77-83. [CrossRef]

18. Korea Disease Control and Prevention Agency. Available online: https://health.cdc.go.kr/healthinfo/biz/pblcVis/main.do (accessed on 8 April 2021).

19. Park, H.; Kim, K. Association of Perceived Stress with Atopic Dermatitis in Adults: A Population-Based Study in Korea. Int. J. Environ. Res. Public Health 2016, 13, 760. [CrossRef]

20. Han, K.M.; Ko, Y.H.; Shin, C.; Lee, J.H.; Choi, J.; Kwon, D.Y.; Yoon, H.K.; Han, C.; Kim, Y.K. Tinnitus, depression, and suicidal ideation in adults: A nationally representative general population sample. J. Psychiatr. Res. 2018, 98, 124-132. [CrossRef]

21. Ministry of Health and Welfare. The Korean National Health and Nutrition Examination Survey (KNHANES). Available online: http:/ / knhanes.cdc.go.kr (accessed on 10 August 2021).

22. Park, K.H.; Cho, J.S.; Lee, K.H.; Shin, S.Y.; Moon, J.H.; Cha, C.I. Rhinoconjunctivitis Quality of Life Questionnaire (RQLQ) as an Evaluator of Perennial Allergic Rhinitis Patients-The First Report. Korean J. Otolaryngol.-Head Neck Surg. 2002, 45, $254-262$.

23. Hayes, A.F.; Cai, L. Using heteroskedasticity-consistent standard error estimators in OLS regression: An introduction and software implementation. Behav. Res. Methods 2007, 39, 709-722. [CrossRef] [PubMed]

24. Juniper, E.F. Quality of life questionnaires: Does statistically significant = clinically important? J. Allergy Clin. Immunol. 1998, 102, 16-17. [CrossRef]

25. Kimata, H. Listening to mozart reduces allergic skin wheal responses and in vitro allergen-specific IgE production in atopic dermatitis patients with latex allergy. Behav. Med. 2003, 29, 15-19. [CrossRef] [PubMed] 
26. Levenstein, S.; Prantera, C.; Varvo, V.; Scribano, M.L.; Berto, E.; Luzi, C.; Andreoli, A. Development of the Perceived Stress Questionnaire: A new tool for psychosomatic research. J. Psychosom. Res. 1993, 37, 19-32. [CrossRef]

27. Elo, A.L.; Leppanen, A.; Jahkola, A. Validity of a single-item measure of stress symptoms. Scand. J. Work Environ. Health 2003, 29, 444-451. [CrossRef] [PubMed]

28. Wamboldt, M.Z.; Laudenslager, M.; Wamboldt, F.S.; Kelsay, K.; Hewitt, J. Adolescents with atopic disorders have an attenuated cortisol response to laboratory stress. J. Allergy Clin. Immunol. 2003, 111, 509-514. [CrossRef]

29. Rod, N.H.; Kristensen, T.S.; Lange, P.; Prescott, E.; Diderichsen, F. Perceived stress and risk of adult-onset asthma and other atopic disorders: A longitudinal cohort study. Allergy 2012, 67, 1408-1414. [CrossRef] [PubMed]

30. Wilson, M.E.; Megel, M.E.; Fredrichs, A.M.; McLaughlin, P. Physiologic and behavioral responses to stress, temperament, and incidence of infection and atopic disorders in the first year of life: A pilot study. J. Pediatr. Nurs. 2003, 18, 257-266. [CrossRef]

31. Wright, R.J. Stress and atopic disorders. J. Allergy Clin. Immunol. 2005, 116, 1301-1306. [CrossRef]

32. Kim, M.B.; Kim, J.H.; Lee, K.H.; Hong, S.C.; Lee, H.S.; Kang, J.W. The association between the parenting stress of the mother and the incidence of allergic rhinitis in their children. Int. J. Pediatr. Otorhinolaryngol. 2017, 101, 37-40. [CrossRef]

33. Andersson, N.W.; Hansen, M.V.; Larsen, A.D.; Hougaard, K.S.; Kolstad, H.A.; Schlunssen, V. Prenatal maternal stress and atopic diseases in the child: A systematic review of observational human studies. Allergy 2016, 71, 15-26. [CrossRef] [PubMed] 